OITS 586

October 1995

\title{
CHAOTIC BEHAVIOR OF PARTICLE PRODUCTION IN BRANCHING PROCESSES
}

\author{
Zhen CAO and Rudolph C. HWA \\ Institute of Theoretical Science and Department of Physics \\ University of Oregon, Eugene, OR 97403
}

\begin{abstract}
The notion of chaotic behavior is examined for particle production in branching processes. Two types of branching are considered: non-Abelian gauge interaction and an Abelian cascade model. Properties of the production processes are investigated by Monte Carlo stimulation. The "temporal" behavior is studied by following the fluctuations in the multiplicities of each generation as the branching evolves. The "spatial" behavior is described in terms of the fluctuations of the normalized factorial moments from event to event. The information dimension and a new entropy index are determined. When all the measures are taken together, they collectively give a strong suggestion that the QCD branching process is chaotic, while the Abelian cascade model is not.
\end{abstract}

\section{Introduction}

Recently we have reported on the results of an investigation in the possible signatures of chaos in branching processes [1]. The aim is to determine whether the nonlinear, non-Abelian dynamics of the quantum Yang-Mills field possesses chaotic behavior. Since in such a dynamical system the number of degrees of freedom increases with time evolution, when the notion of time is not even well defined in the branching process, new measures of trajectory, distance, entropy, etc. must be introduced. We have found that the perturbative QCD branching shows signs indicative of chaos, whereas a model lacking the characteristics of QCD does not. In this paper we give the details of our study.

In the case of classical non-Abelian dynamics special simplifying conditions that reduce the equations of motion to manageable size have been considered and chaotic 
solutions have been found [2, 3]. A more complete investigation of the gauge equations has to be done on the lattice, and it has been shown that the classical non-Abelian gauge theory generally exhibits deterministic chaos, whose Lyapunov exponents can be numerically computed [4-6].

The extension of the above classical problem to the quantum theory of Yang-Mills field is extremely difficult. The current state of knowledge about quantum chaos hovers round semi-classical problems in which classical trajectories are generalized to waves [7, 8]. That is totally inadequate for treating quantum fields, the proliferation of which in a collision process involves issues that are untouched in solving wave equations. The first step toward formulating a feasible program to attack the problem is to dissociate the complications of nonperturbative QCD from the quantum dynamics of non-Abelian fields. In the perturbative domain where the QCD coupling is small, the nature of nonlinear, non-Abelian dynamics is fully present so that the signature of chaotic behavior in gauge theory should nevertheless show up in hard processes. That is an important step of simplification that renders the problem manageable. More specifically, one can narrow the scope and focus on branching processes with QCD splitting functions. The issue then becomes the search for measures that can reveal chaoticity in branching processes.

In such a search it is necessary to keep in mind the special features of QCD. There are many processes that involve branching in real time, such as cell division in biological systems. The emission of photons by an accelerated charge can be regarded as a branching process with sequential ordering. However, for gluons reproducing gluons in the pure gauge theory, the time variable plays no role in the description of a state with $n$ gluons whose momenta are precisely specified. In multiparticle production one works with momenta (or their variants such as rapidity), since they are what can be measured in a collision process. Then in the momentum space the concept of trajectory for a system of increasing number of gluons becomes ill defined. Without trajectories the notion of distance between trajectories is untenable, and the conventional method of examining chaos in classical nonlinear problems is inapplicable. It will therefore be necessary for us to look for other quantities that can describe the difference between states, which evolve from what correspond to nearby initial conditions in the classical problem.

The major difficulty with the study of chaotic behavior of nonlinear gauge dynamics is that the gauge fields are not directly measurable and that the branching processes cannot be tracked experimentally. In collisions at high energies, where the question of chaos in gauge dynamics arises, only the particles in the final states can be measured. Thus to verify any theoretical predictions about chaotic behavior, the loss of information at the end of the branching process must be quantified and presented in a form suitable for experimental determination. To that end we shall introduce an entropy index $\mu_{q}$, which is measurable, and describes the degree of fluctuation of the final particles from event to event.

Before going into details, it may be helpful to state the general idea underlying 
the work. In classical dynamics if the coordinates $q_{\alpha}(t)$ and momenta $p_{\alpha}(t)$ specify the system, then the trajectory in phase space is well defined in the familiar way. In classical field theory the fields, $E_{\alpha}(x, t)$ and $B_{\alpha}(x, t)$ say, form a field configuration defined over all space $x$ at any given time, and the change of the configuration as time evolves specifies a trajectory in a generalized sense. For quantized fields one works in the Fock space so that the number of quanta becomes a variable that specifies an important aspect of the state of the system. The distance between two trajectories in that case must therefore involve, among other quantities, the difference between the numbers of quanta in the system. Thus in particle production at high energy where the multiplicity $n$ of produced particles is an essential observable, the fluctuation of $n$ from event to event must be viewed as the consequence of swings of trajectories that have almost the same initial condition. With that connection in mind it is natural to generalize the conventional treatment of nonlinear dynamics to an approach that places emphasis on tracking the multiplicity fluctuations from event to event in high energy collisions. After describing the QCD dynamics of branching and a simple $\chi$ model in Sec. 2, we treat the temporal behavior of the branching processes in Sec. 3 . Then in Secs. 4 and 5 we consider the spatial properties of the final state. Concluding remarks are given at the end.

\section{Gluon Branching and the $\chi$ Model}

To carry out this investigation it is necessary to use computer simulation to generate events of particle production through branching. Only then is it possible to study the nature of fluctuations of the final-state particles. For the simulation we shall use algorithms based on two opposite types of dynamics. One is, of course, the QCD dynamics. For simplicity we shall focus on only the pure gauge theory without quarks. At the end of the evolution we shall identify the partons as particles to avoid the complication of hadronization, which is inessential to the question of whether the gluon branching dynamics is chaotic. The other is a cascade model, to be called the $\chi$ model, which has none of the features of the QCD; in particular, it does not have infrared and collinear divergences. It is studied in order to provide a contrast to the gauge theory so that our measures for chaoticity can be tested on these two contrasting branching dynamics.

In both branching processes the initial parton has virtuality $Q^{2}$, and successive branchings continue until the virtualities of all partons are $\leq Q_{0}^{2}$. In pure-gauge QCD the splitting function at each vertex of branching is (for $g \rightarrow g g$ )

$$
P(z)=6\left[\frac{1-z}{z}+\frac{z}{1-z}+z(1-z)\right],
$$

where $z$ is the momentum fraction of the daughter parton in the frame where the mother parton's momentum is 1 . In the $\chi$ model we keep only the last term of (11)

$$
P(z)=6 z(1-z)
$$


so that it has no divergences at $z=0$ and 1 . The presence of those divergences is, of course, the source of complication for QCD that must be treated carefully.

We follow Odorico's procedure [9] to develop the algorithm for Monte Carlo simulation of parton shower in QCD. Because of the soft gluon and collinear divergences many partons are emitted at small $z$ and small angles but are not resolvable. The probability that a parton at $q^{2}=Q^{2}$ can survive without emitting a resolvable parton until $q^{2}=Q_{0}^{2}$ is the Sudakov form factor [9, 10]

$$
\Delta\left(Q, Q_{0}^{2}\right)=\exp \left[-\int_{Q_{0}^{2}}^{Q^{2}} \frac{d t^{\prime}}{t^{\prime}} \frac{\alpha_{s}\left(t^{\prime}\right)}{2 \pi} \int_{z_{0}\left(t^{\prime}\right)}^{1-z_{0}\left(t^{\prime}\right)} d z P(z)\right],
$$

where the limits of the $z$ integration define what is meant by resolvable. The first step of the simulation is to set $z_{0}=Q_{0}^{2} / Q^{2}$ and calculate from $\Delta\left(Q^{2}, Q_{0}^{2}\right)$ whether a parton starting from $Q^{2}$ evolves to $Q_{0}^{2}$ without branching. If not, then the value of $q^{2}$ at which the branching occurs is determined by solving

$$
\frac{1-\Delta\left(Q^{2}, Q_{0}^{2}\right) / \Delta\left(q^{2}, Q_{0}^{2}\right)}{1-\Delta\left(Q^{2}, Q_{0}^{2}\right)}=R_{\#}
$$

where $R_{\#}$ is a random number between 0 and 1 . With that $q^{2}$, the value of $z$ is then generated in accordance to the distribution $P(z)$ given in (1) keeping only what is in the range $Q_{0}^{2} / q^{2} \leq z \leq 1-Q_{0}^{2} / q^{2}$. With that $z$, the daughter partons are assigned the maximum virtualities $t_{1}=z q^{2}$ and $t_{2}=(1-z) q^{2}$, from which further evolutions are carried out by repeating the above procedure starting with $t_{1}$ and $t_{2}$. When the virtuality of a descendant reaches $q^{2} \leq Q_{0}^{2}$, then that branch of the tree terminates. We shall put $Q_{0}=1 \mathrm{GeV}$, to be definite. In the simulation we shall set $N_{c}=3$, $N_{f}=0$ so that

$$
\alpha_{s}\left(q^{2}\right)=4 \pi / 11 \log \left(q^{2} / \Lambda^{2}\right)
$$

where $\Lambda^{2}$ will be set at $250 \mathrm{MeV}$.

We have also tried to simulate the parton shower using the algorithm of Weber [10] and found that the result differs very little from that based on the above algorithm of Odorico [9] for the type of measures we calculate. Since Odorico's method is far more efficient, we have chosen to use it throughout this work.

In the $\chi$ model, because of no infrared and collinear divergences, there is no automatic $q^{2}$ evolution. However, in order to compare its properties with those of QCD, we introduce by hand $Q^{2}$ dependence of the branching process by requiring that at each vertex the two daughter partons have virtualities $z q^{2}$ and $(1-z) q^{2}$, when the mother virtuality is $q^{2}$, and $z$ is generated by using (2) for $0 \leq z \leq 1$. As with QCD, we require branching to continue successively until the virtualities of all partons become $\leq Q_{0}^{2}$. Since $q^{2}$ is not degraded along a parton line, there are far more particles produced in the $\chi$ model than in QCD for the same $Q^{2}$, but that is immaterial, since our measure of chaoticity will not depend on $Q / Q_{0}$. Clearly, the dynamics of branching in the $\chi$ model is very different from QCD. We use it to exemplify the Abelian dynamics that has no infrared and collinear divergences. 


\section{Temporal Behaviors of Branching}

Each branching process can be represented by a tree diagram, since recombination of partons is not considered. The vertices of the tree could be ordered vertically in accordance to the values of $q^{2}$ of the mother partons. Then there are many diagrams with the same topology that can describe different evolution processes leading to the same number of particles at the end, but belonging to different final states. If we defer the considerations of the momenta of the final particles until the next section, we can simplify the problem by focusing on only the topology of the tree diagram. In that way we study the fluctuation of the particle multiplicity at the expense of ignoring their momenta. What is gained is the possibility of defining a trajectory in the multiplicity space for a branching process.

In emphasizing the topology of a diagram let us draw all diagrams in such a way that all partons of the same generation are placed at the same level regardless of their $q^{2}$. An example is shown in Fig. 1. Where the partons are horizontally has no significance in that diagram. Vertically, the branching points of the same generation are placed at the same level. All partons that reach the final state (represented by the dashed line) have $q^{2} \leq Q_{0}^{2}$. No information concerning $q^{2}>Q_{0}^{2}$ is carried by the vertical position of the lines in the diagram. Let $i$ denote the generation, staring with $i=0$ for the initial parton at $q^{2}=Q^{2}$. Let $b_{i}$ denote the number of branching points at the $i$ th generation. In Fig. 1 we have $b_{i}=1,2,3,3,1$ for $i=0, \cdots, 4$. We can define a vector $\mathbf{b}=\left(b_{0}, b_{1}, \cdots\right)$ with as many components as there are nonvanishing $b_{i}$. Thus for the diagram in Fig. 1 we have $\mathbf{b}=(1,2,3,3,1)$. If this description were to be applied to an electron radiating photons, then a bremsstrahlung diagram would have $\mathbf{b}=(1,1,1, \cdots)$. On the other hand, for cell reproduction where every cell subdivides into two, then $\mathbf{b}=(1,2,4,8, \cdots)$. The description can be further simplified, if we define

$$
x_{i}=\log _{2} b_{i}
$$

and the corresponding vector $\mathbf{x}=\left(x_{0}, x_{1}, \cdots\right)$. Since the minimum $x_{i}$ is 0 and the maximum $x_{i}$ is $i$, the two extreme vectors are $\mathbf{x}=(0,0,0, \cdots)$ which is like bremsstrahlung, and $\mathbf{x}=(0,1,2,3, \cdots)$ which is like cell-reproduction. They are shown in Fig. 2 by the thick lines, where $x_{i}$ is plotted against $i$. All possible tree diagrams of branching processes are represented by a line in between the two boundary (thick) lines, as illustrated by the thin line in Fig. 2. Such a line specifies a trajectory.

For finite $Q^{2}$ the branching cannot go without end, so there is always a maximum $i$, which we denote by $i_{\max }$. The value of $i_{\max }$ varies from event to event, even if $Q^{2}$ is fixed for all events. Two paths with the same $i_{\max }$ may differ for $0<i<i_{\max }$, and the total numbers of partons produced would be different. In general, for any two trajectories $\mathbf{x}$ and $\mathbf{x}^{\prime}$ one can define $d_{i}=\left|x_{i}-x_{i}^{\prime}\right|$ at every $i$ where $x_{i}$ and $x_{i}^{\prime}$ exist simultaneously. Obviously, $d_{i}$ can be regarded as the distance between the two trajectories. Since two trajectories in $x$ space can cross, $d_{i}$ may vanish at nonzero $i$. 
Since $i$ is an integer, $x_{i}$ has discrete values. Thus the trajectories cannot be dense in the continuum space of $i$ and $x_{i}$, but for the discrete support of the trajectories we may regard the collection of all possible trajectories as being dense in the sense that a trajectory can pass through any allowed value of $\left(i, x_{i}\right)$.

To relate the description even more closely to the classical treatment of chaos, we now address the question of sensitivity to initial condition. In classical nonlinear dynamics one can specify the point in phase space where a trajectory begins and vary that point $n$ in as small a neighborhood $N_{\varepsilon}$ as one chooses. In non-Abelian gauge dynamics or in the $\chi$ model, the initial condition is that the initial parton has virtuality $q^{2}=Q^{2}$. Being quantum mechanical, it is not necessary to vary $q^{2}$ in a small neighborhood of $Q^{2}$. Quantum fluctuation is sufficient to guarantee that the final state of the branching process will vary from event to event, even if all events start out at precisely the same $Q^{2}$. Thus the relationship to the classical consideration of initial condition is as follows. Consider $\mathcal{N}$ random points in the neighborhood $N_{\varepsilon}$ of the initial point in phase space of a classical trajectory. Any such point can be mapped to the beginning of a particular branching process. A set of $\mathcal{N}$ events in the Monte Carlo simulation all starting out at the same $Q^{2}$ then correspond to $\mathcal{N}$ trajectories beginning in the neighborhood $N_{\varepsilon}$ in the classical case. With this correspondence between the two problems it is then sensible to suggest that instead of studying the distance between two neighboring trajectories, one should consider all $\mathcal{N}$ events in the Monte Carlo simulation and examine the mean deviation from the average of the parton multiplicities. Indeed, the study of fluctuations of observable quantities will be the main theme of our approach to analyzing chaotic behavior of branching processes.

In Fig. 3 we show schematically several possible trajectories of the branching processes, all started out at the same $Q^{2}$. Not only can trajectories that have the same $i_{\max }$ be different, those with different $i_{\max }$ are even more dissimilar. For the purpose of studying "temporal" behavior, which corresponds to the dependence on the generation $i$ in the branching process, only trajectories with the same $i_{\max }$ can be compared. Thus it is necessary to know the distribution $P\left(i_{\max }\right)$ of $i_{\max }$ for all events. Fig. 4 shows the result of $10^{5}$ simulations for each of the two type of branching processes discussed in the previous section. The initial virtualities are such that $Q / Q_{0}=10^{3}$ for QCD and 15 for the $\chi$ model. These values are chosen so that the average multiplicities are comparable, as we shall show below. Both $P\left(i_{\max }\right)$ are Gaussian-like distributions, with the width being larger for QCD. This is the first indication of more fluctuation for QCD as compared to the $\chi$ model. In choosing a narrow band of $i_{\max }$ for further investigation, we select the shaded regions in Fig. 4 that are situated at the maximum of $P\left(i_{\max }\right)$.

As mentioned before, time is not a well-defined variable in the branching process. It is deemphasized when we focus on the topololgy of the tree diagrams. The generation label $i$ carries only a rough notion of time, since it is well known, as in family genealogy, that a son can be younger than a grandson. However, there is one quantity 
that increases monotonically with $i$; it is the parton multiplicity $n_{i}$

$$
n_{i}=1+\sum_{j=0}^{i-1} b_{j}=1+\sum_{j=0}^{i-1} 2^{x_{j}}
$$

even though $x_{i}$ may rise and fall with $i$. Define the average parton multiplicity $\left\langle n_{i}\right\rangle$ at generation $i$ by

$$
\left\langle n_{i}\right\rangle=\frac{1}{\mathcal{N}} \sum_{e=1}^{\mathcal{N}} n_{i}^{e}\left(i_{\text {max }}\right)
$$

where $n_{i}^{e}$ is the value of $n_{i}$ at $i$ for the eth event and the sum is over all $\mathcal{N}$ events having the same $i_{\max }$ chosen. This quantity $\left\langle n_{i}\right\rangle$ may be taken to play the role of time in the branching process, although no linear dependence is implied. In Fig. 5 we show the simulated results on $\left\langle n_{i}\right\rangle$ as functions of $i$ for various values of $Q / Q_{0}$. In both types of branching processes $\left\langle n_{i}\right\rangle$ increases monotonically with $i$, the rate being fastest at midrange of $i$. The values of $Q / Q_{0}$ are chosen such that $\left\langle n_{i}\right\rangle$ are roughly in the same range for the two types.

For the measure that describes the fluctuation of the trajectories, and therefore of the multiplicities, we use the normalized variance

$$
V_{i}=\frac{\left\langle n_{i}^{2}\right\rangle-\left\langle n_{i}\right\rangle^{2}}{\left\langle n_{i}\right\rangle^{2}},
$$

which clearly gives a measure of the average "distance" between trajectories. It is always positive, as a distance function should be. In Fig. 6 is shown the results of simulation for the two cases. The sustained increase in the lower half of $i$ range for QCD is a distinctive feature that is not shared by the $\chi$ model.

A more transparent way of presenting this result is to plot $V_{i}$ vs $\left\langle n_{i}\right\rangle$, as shown in Fig. 7. It corresponds to plotting the distance $d(t)$ against $t$ in the classical problem, except that here $\left\langle n_{i}\right\rangle$ is not exactly $t$, but some representation of it. Now, the behavior for QCD appears universal, i.e., independent of $Q / Q_{0}$ over a wide range. In the loglog plot the behavior is approximately linear. The same is not true for the $\chi$ model: $V_{i}$ increases initially, then drops precipitously, as $\left\langle n_{i}\right\rangle$ is increased. Furthermore, the maximum decreases with increasing $Q / Q_{0}$. Thus the uncertainty in the parton multiplicity increases with $\left\langle n_{i}\right\rangle$ in the former case, but saturates and then decreases in the latter case. If that uncertainty can be regarded as a measure of chaos, then the QCD dynamics is chaotic, while the $\chi$ model is not. We can express the dependence of $V_{i}$ on $\left\langle n_{i}\right\rangle$ in the QCD case in the form of a power law

$$
V_{i} \propto\left\langle n_{i}\right\rangle^{\kappa}
$$

Then from Fig. 7 we find $\kappa$ to be $\simeq 0.4$. Writing (10) in the form of an exponential

$$
V_{i} \propto \exp \left(\kappa \ln \left\langle n_{i}\right\rangle\right) \quad,
$$


we may compare it to the definition of Lyapunov exponent $\lambda$ in $d(t) \sim e^{\lambda t}$. If $\ln \left\langle n_{i}\right\rangle$ can be interpreted to correspond to $t$, then $\kappa$ may be said to play the role of the Lyapunov exponent. However, nothing firm can be proven about these correspondences, so the above remarks should only be taken as a possible orientation in interpreting the implication of the result obtained.

From what has so far been done, it is not possible for us to find a criterion on the magnitude of $\kappa$ to signify robust chaoticity. Furthermore, $\left\langle n_{i}\right\rangle$ and $V_{i}$ are aspects of the parton state at generation $i$ before the completion of the branching processes. Thus they are not experimentally measurable. For particle production in high energy collisions, the temporal behavior of branching is primarily a theoretical problem. For experimental verification of any theoretical prediction, it is necessary to focus on the final state, which is the subject of the next two sections.

\section{Factorial Moments, Entropy, and Information Dimension}

In the final state the complete information about the partons, now identified as observable particles, is registered by their momenta. If the branching trajectories never change, then there is certainty in where to find those particles in the momentum space, and the entropy (to be defined below) is zero, corresponding to no loss of information. However, quantum fluctuation alone is sufficient to cause fluctuation in the final-state momenta, so entropy is not expected to vanish. In multifractal analysis of complex patterns, the information dimension is a compact way of summarizing the dependence of the entropy on resolution scale [11]. Since multifractal analysis of the multiplicity fluctuation in high-energy collisions has been proposed [12, 13] and carried out [14, 15], it is natural to examine the information dimension for the branching processes considered here

If $p_{j}$ denotes the fraction of particles in an event that fall into the $j$ th bin of size $\delta$, then in the limit of many such bins in the system under study, the entropy is defined by 13

$$
S=-\sum_{j} p_{j} \ln p_{j}
$$

and the information dimension $D_{1}$ is 16

$$
D_{1}=-\lim _{\delta \rightarrow 0} S / \ln \delta
$$

The study of self-similar behavior of particle production has been greatly facilitated by the use of normalized factorial momenta $F_{q}$, suggested by Białas and Peschanski [17], who first showed that the statistical fluctuations are filtered out by those moments. However, since $F_{q}$ is defined for integer $q \geq 2$, it cannot be used to determine the 
information dimension, which involves the derivative of $F_{q}$ with repect to $q$ at $q=1$. For that reason the $G_{q}$ moments for all real $q$, positive and negative, were introduced for analyzing the multifractal structure of particle production data [12, 13, 15]. Their drawback is that the statistical fluctuations must be taken out of $G_{q}$ explicitly by hand. More recently, a method for continuing $F_{q}$ to noninteger $q$, while maintaining its virtue of not being contaminated by statistical fluctuations, has been developed [18]. We shall therefore make use of it to determine $D_{1}$.

Before describing the details of the calculations, let us first make clear the space in which the self-similar behavior is examined. With $z_{i}$ denoting the momentum fraction of an ith generation daughter parton at a branching vertex, where the mother parton has momentum 1, the momentum fraction of a final particle is then

$$
x=\prod_{i} z_{i}
$$

where the product is taken over all generations of a particular path in the branching tree, leading from the initial parton to the final particle under consideration. Since all $z_{i}$ are known in a simulated event, the values of $x$ for all particles can be calculated; they are all crowded in the $x=0$ region, since their sum is 1 . This is especially true for QCD branching because of the soft gluon divergence in (11). If we expand the $x=0$ region by using the variable $\zeta=-\log _{10} x$, we get a broad Gaussian-like distribution, as shown is Fig. 8, with the peak at $\zeta=3$ corresponding to $x=10^{-3}$. The situation is not as bad for the $\chi$ model, but still most particles are found in the small $x$ region, as shown in Fig. 9. This highly uneven distribution of $x$ is inappropriate for data analysis that involves the partition of the space into small bins.

One can define another variable $X$, in terms of which the distribution is much smoother [19]. Let the inclusive distribution in $x$, averaged over many events, be $\rho(x)$. Then the cumulative variable $X$ is defined by

$$
X(x)=\int_{x_{1}}^{x} \rho\left(x^{\prime}\right) d x^{\prime} / \int_{x_{1}}^{x_{2}} \rho\left(x^{\prime}\right) d x^{\prime}
$$

where $x_{1}$ and $x_{2}$ are two extreme points in the distribution $\rho(x)$, between which $X$ varies from 0 to 1 . In terms of $X$ the inclusive distribution $\rho(X)$ is constant. For QCD branching we shall take $\rho\left(x^{\prime}\right)$ to be the $\zeta$ distribution as in Fig. 8; for the $\chi$ model $\rho\left(x^{\prime}\right)$ will be just the $x$ distribution as in Fig. 9. An illustration of the $\rho(X)$ distribution for QCD is shown is Fig. 10 after simulating $5 \times 10^{4}$ events at $Q / Q_{0}=10^{3}$. Note the expanded scale along the vertical axis.

Now, in $X$ space we divide the interval $0 \leq X \leq 1$ into $M$ bins of width $\delta=1 / M$. Let $n_{j}$ be the number of final particles in the $j$ th bin in any given event. The factorial moment of $q$ th order is

$$
f_{q}(M)=M^{-1} \sum_{j=1}^{M} n_{j}\left(n_{j}-1\right) \cdots\left(n_{j}-q+1\right)
$$


The normalized factorial moment after averaging over all events is

$$
F_{q}=\left\langle f_{q}\right\rangle /\left\langle f_{1}\right\rangle^{q}
$$

Since $\left\langle f_{q}\right\rangle$ is the average of $n_{j} ! /\left(n_{j}-q\right)$ ! over all bins and over all events, we may write it as

$$
\left\langle f_{q}\right\rangle=\sum_{n=q}^{\infty} \frac{n !}{(n-q) !} P_{n}
$$

where $P_{n}$ is the multiplicity distribution in a bin. In general, $P_{n}$ may be expressed as a convolution of the statistical $(S)$ and the dynamical $(D)$ contributions to the multiplicity fluctuations:

$$
P_{n}=S \otimes D=\int_{0}^{\infty} d t \frac{t^{n}}{n !} e^{-t} D(t)
$$

where a Poissonian distribution has been used for $S$. Putting (19) into (18) yields

$$
\left\langle f_{q}\right\rangle=\int_{0}^{\infty} d t t^{q} D(t)
$$

This is the standard moment of the dynamical distribution that has no statistical contamination [17]. If $D(t)=\delta(t-\bar{n})$, so that $P_{n}$ is Poissonian, then $\left\langle f_{q}\right\rangle=\bar{n}^{q}$ and $F_{q}=1$. Any deviation of $F_{q}$ from 1 is an indication of the presence of dynamical fluctuation.

Phenomenologically, one does not have access to $D(t)$. Using $P_{n}$ as input from experiment or from simulation, one can determine $\left\langle f_{q}\right\rangle$, but only for integer $q$. One may replace the factorials in (18) by gamma functions in order to continue (18) to noninteger $q$ [20]. But that procedure does not result in $F_{q}=1$ for Poisson distribution for all $q$. In Ref. 18 a continuation procedure is developed that guarantees $F_{q}=1$ for all $q$ when $D(t)$ is trivial. That method will not be described here, but will be used to determine $F_{q}$ for our branching processes.

For notational brevity let us write the RHS of $(20)$ as $\left\langle t^{q}\right\rangle_{D}$. Then we have

$$
F_{q}=\left\langle\left(t /\langle t\rangle_{D}\right)^{q}\right\rangle_{D}=\left\langle(p M)^{q}\right\rangle_{D}
$$

where $p$ is the fraction of event multiplicity in a bin, as in (12). Thus from (12) we get

$$
S=-M\langle p \ln p\rangle_{D}=\ln M-\left.\frac{d}{d q} \ln F_{q}\right|_{q=1} .
$$

If $F_{q}$ has a power-law behavior near $q=1$, i.e.,

$$
F_{q} \propto M^{\varphi_{q}}
$$


it then follows from (13) that in the large $M$ limit

$$
D_{1}=1-\left.\frac{d \varphi_{q}}{d q}\right|_{q=1}
$$

Note that this relationship is derived under the assumption that (22) is meaningful, which demands that $F_{q}$ is well defined in a range of $q$ around $q=1$ and that $d F_{q} / d q$ contains only dynamical information without spurious contribution arising from improper continuation procedure [18].

If dynamically the bin multiplicity is evenly distributed among all $M$ bins so that $D(t)=\delta\left(t-t_{0}\right)$, then $F_{q}=1$ for all $q$ (including non-integer $q$ ) and (22) gives $S=\ln M$. On the other hand, if dynamically only a fraction $a$ of the $M$ bins are populated, the rest empty, i.e.

$$
D(t)=a \delta\left(t-t_{1}\right)+(1-a) \delta(t),
$$

then $\left\langle f_{q}\right\rangle=a t_{1}^{q}$ and $F_{q}=a^{1-q}$ for all $q$. Hence, (22) yields $S=\ln (a M)$. If, in particular, only one bin is non-empty, i.e., $a=1 / M$, then $S=0$. Thus (25) offers a simple way of seeing how the entropy increases from 0 to $\ln M$, as the fraction of nonempty bins increases from $1 / M$ to 1 . If there is only one non-empty bin, the dynamics is like one with a classical trajectory that has no dispersion in where the particles are to be found in the momentum space. Without loss of information the entropy is zero. If there is maximum dispersion corresponding to all bins being equally likely to be populated, then the entropy is maximum.

We now apply this method of analysis to our simulated branching processes. First, we show in Fig. 11 the multiplicity distributions in QCD for various bin sizes $(\delta=$ $1 / M)$ after $10^{5}$ events. It should be understood that Fig. 10 shows the inclusive $X$ distribution after summing over all events. But event by event the fluctuation in $X$ space is much greater. Upon dividing the interval $0 \leq X \leq 1$ into $M$ bins and counting the multiplicity in each bin, one then obtains $P_{n}(M)$ after averaging over all the events. The degree of fluctuation around the decreasing $\langle n\rangle$, relative to that average, as $M$ is increased, is what is measured by the normalized factorial moments $F_{q}$ defined by (17) and (18). This is unlike our theoretical calculation based on (20) and (25) because $D(t)$ is not known explicitly in the simulation. The results are shown in Fig. 12 for both QCD and $\chi$ model. Since $F_{q=1}=1$ by definition, we see that $F_{q}$ increases with $q$ in QCD, but decreases with increasing $q$ in the $\chi$ model. It means that the distributions $P_{n}(M)$ for QCD are wider than Poisson, but for the $\chi$ model they are sub-Poissonian. Fig. 12 also shows that there is very little $M$ dependence in either case. For non-integer $q$ values a substantial amount of work is needed to continue $F_{q}$ according to the method of [18]. The result is shown in Fig. 13. It is clear that for $q>1$ all $F_{q}$ are greater in QCD than in the $\chi$ model. It corresponds to more fluctuations in QCD than in the $\chi$ model, a result that is consistent with the finding of the previous section. 
It is also evident from Fig. 13 that $d F_{q} /\left.d q\right|_{q=1}$ is essentially independent of $M$ in both cases. Whereas $S$ is still dependent on $M$ according to (22), the $M$ independence

of $d \ln F_{q} /\left.d q\right|_{q=1}$ requires, on account of (23), that $d \varphi_{q} /\left.d q\right|_{q=1}=0$. This together with (24) entails

$$
D_{1}=1
$$

in both cases. Thus there is no interesting multifractal property. This particular approach to finding a useful signature of chaotic behavior ends in failure.

The smoothing of the phase space by use of the $X$ variable in place of the momentum fraction variable $x$ has undoubtedly contributed to eliminating some aspect of the fractal structure. But what has not been removed is the more interesting consequences of the dynamics. The triviality of $D_{1}$ states only that there is no nontrivial self-similarity in the fluctuations measured. The latter are quantified by $F_{q}$, which involves both the bin and event averages of the factorial product in (16). The result suggests that some of the fluctuations have been lost by the averaging process. In the next section we develop a scheme to recapture that which has been lost.

\section{$5 \quad$ Entropy Index}

We know that event by event there are large fluctuations in where the produced particles are in the $X$ space. What we want to do now is to find a way to register the fluctuation (from event to event) of the fluctuation (in the $X$ distribution).

Let $F_{q}^{e}$ denote the normalized factorial moment of the eth event:

$$
F_{q}^{e}=f_{q}^{e} /\left(f_{1}^{e}\right)^{q}
$$

where $f_{q}^{e}$ is as defined in (16) for the eth event. Clearly, $F_{q}^{e}$ changes with $e$ for fixed $q$ and $M$. In Fig. 14 we show the distribution $P\left(F_{q}\right)$ after $10^{5}$ simulated events of QCD branching, the label $e$ being omitted. For clarity, only $q=2$ and 3 are exhibited for a wide range of $M$ values. At small $M$ the distribution has a peak at $F_{q} \approx 1$. Indeed, if $M=1$ and event multiplicity is large, then $F_{q}$ should be very nearly 1 for all events. At large $M$ a peak at $F_{q}=0$ is developed, especially at high $q$, because the bin multiplicity for small $\delta$ is small and may in many events be less than $q$ for all bins, in which cases $F_{q}^{e}=0$. This large fluctuation in $P\left(F_{q}\right)$ is what we want to capture, and would be lost if $F_{q}$ is averaged over all events. Clearly we should take the moments of $F_{q}$ before performing the average.

We may think of $F_{q}^{e}$ as the horizontal moments in the $X$ space, and then define the vertical moments in the event space as follows:

$$
\left\langle F_{q}^{p}\right\rangle=\frac{1}{\mathcal{N}} \sum_{e=1}^{\mathcal{N}}\left(F_{q}^{e}\right)^{p}
$$


where $\mathcal{N}$ is the total number of events and $p$ is a positive real number not restricted to integers. The normalized moments of moments are

$$
C_{p, q}(M)=\left\langle F_{q}^{p}(M)\right\rangle /\left\langle F_{q}(M)\right\rangle^{p}
$$

If $C_{p, q}(M)$ has a power-law behavior in $M$, i.e.,

$$
C_{p, q}(M) \propto M^{\psi_{q}(p)}
$$

then we can define

$$
\mu_{q}=\left.\frac{d}{d p} \psi_{q}(p)\right|_{p=1}
$$

which will be referred to as the entropy index.

To see the connection between $\mu_{q}$ and entropy, let us define

$$
P_{q}^{e}=F_{q}^{e} / \sum_{e=1}^{\mathcal{N}} F_{q}^{e}
$$

and a (new) entropy in the event space

$$
S_{q}=-\sum_{e=1}^{\mathcal{N}} P_{q}^{e} \ln P_{q}^{e} .
$$

Furthermore, define the moments

$$
H_{p, q}=\sum_{e=1}^{\mathcal{N}}\left(P_{q}^{e}\right)^{p}
$$

which are related to $S_{q}$ by

$$
S_{q}=-\left.\frac{d}{d p} \ln H_{p, q}\right|_{p=1}
$$

On the other hand, $H_{p, q}$ can be related to $C_{p, q}$ by

$$
C_{p, q}=\mathcal{N}^{p-1} H_{p, q}
$$

so we have

$$
\left.\frac{d}{d p} \ln C_{p, q}\right|_{p=1}=\ln \mathcal{N}-S_{q}
$$

In then follows from (30) and (31) that

$$
S_{q}=\ln \left(\mathcal{N} M^{-\mu_{q}}\right)
$$


apart from a possible additive term that is independent of $\mathcal{N}$ and $M$.

This entropy, as defined in (33), is clearly different from the entropy $S$ defined in (12). To emphasize that $S_{q}$ is defined in the event space, we may call it eventropy. We can think of the event space as a one dimensional space with $\mathcal{N}$ sites. At each site we can register a number $F_{q}^{e}$. If $F_{q}^{e}$ is the same at each site, then $P_{q}^{e}=1 / \mathcal{N}$, and $S_{q}=\ln \mathcal{N}$. We should think of this as being highly disordered in the event space, since $F_{q}^{e}$ is spread out uniformly over all space. The larger the number of events, the larger is the eventropy. This is very similar to the situation where $S=\ln M$, when the bin multiplicity is uniformly distributed in the $X$ space that has $M$ bins. A branching dynamic that results in the same $F_{q}^{e}$ for every event does not fluctuate in the branching processes. It corresponds to nearby trajectories staying nearby throughout. In short, the dynamics is not chaotic. For $S_{q}$ to be $\ln \mathcal{N}$, we see from (38) that $\mu_{q}$ must vanish. Thus small $\mu_{q}$ corresponds to large eventropy, which in turn implies no chaotic behavior.

On the other hand, if we consider the other extreme where all $F_{q}^{e}$ are zero except one event $e^{\prime}$, then $P_{q}^{e}=\delta_{e e^{\prime}}$ and $S_{q}=0$. This is highly ordered in the event space, but the fluctuation of $F_{q}^{e}$ from zero to a nonzero value is large. Generally speaking, if the distribution $P\left(F_{q}\right)$ is broad, the fluctuation is large, initially nearby trajectories become widely separated in the final states of different events, and the dynamics is chaotic. In order for the eventropy to be small, $\mu_{q}$ must be large. Thus large entropy index implies chaotic behavior.

In Fig. 15 the results of our simulation are shown for $q=2,3,4$ and $p=0.5,1.0$, 1.5, and 2.0. From the log-log plots one can identify approximate scaling behavior. We used the region $M=5-20$ to determine the value of $\mu_{p}$, for which $C_{p, q}$ for an incremental region of $p$ around $p=1$ has to be examined. We obtain

$$
\begin{array}{rllll}
\mu_{q}^{(\mathrm{QCD})} & =0.0061, & 0.054, & 0.23 & (q=2,3,4) \\
\mu_{q}^{(\chi)} & =0.0014, & 0.010, & 0.046 & (q=2,3,4)
\end{array}
$$

This result is plotted in Fig. 16. Clearly, $\mu_{q}^{(Q C D)}$ is much larger than $\mu_{q}^{(\chi)}$. It is suggestive that the QCD dynamics is chaotic, while the $\chi$ model is not. However, at this point we have no quantitative criterion on how large $\mu_{q}$ must be in order to be chaotic. In classical nonlinear problems there is also no criterion on how long the time lapse must be in order for the distance between nearby trajectories to become sufficiently far apart to qualify for divergent behavior. Nevertheless, the positivity of the Lyapunov exponent is a simple condition, the counterpart of which for the entropy index is lacking.

\section{Conclusion}

We have explored various ways of measuring chaoticity of branching processes. Because the problem is far more complicated than a classical nonlinear problem, the 
search has not been a straightforward extension of the conventional ideas. Our approach has been based on a simple premise: if a branching process is chaotic, then it should be hard to predict what the likely outcome of an event would be, given the knowledge of the result of a previous event. It means that there are large fluctuations from event to event.

To be more concrete, we have focused first on the temporal development of branching. The variance $V_{i}$ of the multiplicities at a particular generation $i$ can be regarded as a measure of the mean distance between "trajectories". We have found that $V_{i}$ increases with $\left\langle n_{i}\right\rangle$ in a power-law fashion for QCD branching, but not for the $\chi$ model, an example of Abelian branching dynamics. That is the first indication that the QCD dynamics has the characteristics of being chaotic. We have not investigated the behavior of the higher moments, $V_{i}$ being related to only the second moment. It is probably safe to predict that the higher moments would increase even more rapidly with $\left\langle n_{i}\right\rangle$.

For fluctuations in the final state where experimental observations are feasible, we have considered $F_{q}, D_{1}, C_{p, q}$, and $\mu_{q}$ as possible measures of chaoticity. It turns out that the information dimension is not effective, but the entropy index can be. What emerges is a realization that one should study the phase space and the event space simultaneously. More specifically, consider a two-dimensional lattice, where the horizontal axis has $M$ sites, corresponding to the $M$ bins in the $X$ space, and where the vertical axis has $\mathcal{N}$ sites, corresponding to the $\mathcal{N}$ events in the event space. For every event there is a horizontal array of $M$ numbers, indicating the particle multiplicities in the $M$ bins. The moments of moments $C_{p, q}$ summarize the distribution on this whole lattice without significant loss of information. The entropy index $\mu_{q}$ extracts from this lattice of numbers a simple numerical quantity that can characterize the degree of fluctuations on the lattice. The larger $\mu_{q}$ is, the more chaotic the system is. Unfortunately, we do not have at this point a criterion on the threshold value of $\mu_{q}$, above which chaotic behavior will definitely occur. Further investigation on this aspect of the problem is undoubtedly warranted.

Each of the features studied reveals some aspect of the chaotic behavior of QCD dynamics. Taken together collectively, they present a strong contrast from the results of the $\chi$ model. One may wonder what the mechanisms are in the branching processes that can lead to such diverse outcomes. Our view is that in QCD branching there is a tension between two opposing forces that is absent in the $\chi$ model. The collinear divergence implies a strong preference for small angle emission of partons in the branching process. But if the emission angle is too small to be resolved, there is no branching. Since evolution without branching is suppressed by the Sudakov form factor, there is a competition between evolution by emitting a resolvable gluon and a preference for emitting a collinear unresolvable gluon. It is this tension that leads to large fluctuations in the number of branching points and hence to chaotic behavior. The $\chi$ model has no collinear divergence and no $q^{2}$ evolution, so there is no tension to cause large fluctuations. What we learn here may lay the foundation for finding ways 
to treat nonperturbative processes, for which chaotic behavior may play a crucial role in the evolution of a large system of quarks and gluons, such as those produced in a heavy-ion collision at high energy.

\section{Acknowledgment}

One of us (RCH) is grateful to C. B. Chiu, K. Geiger, B. L. Hao, S. G. Matinyan, and B. Müller for helpful discussions. This work was supported in part by the U.S. Department of Energy under Grant No. DE-FG06-91ER40637. 


\section{References}

[1] Z. Cao and R. C. Hwa, Phys. Rev. Lett. 75, 1268 (1995).

[2] S.G. Matinyan et. al., Sov. Phys. JETP 53, 421 (1981).

[3] S.G. Matinyan, Sov. J. Part. Nucl. 16, 226 (1985) and other references quoted therein.

[4] B. Müller and A. Trayanov, Phys. Rev. Lett. 68, 3387 (1992).

[5] T.S. Biró, C. Gong, B. Müller and A. Trayanov, Int. J. Mod. Phys. C5, 113 (1994).

[6] C. Gong, Phys. Lett. B298, 257 (1993), Phys. Rev. D 49, 2642 (1994).

[7] M Tabor, Chaos and Integrability of Nonlinear Dynamics (Wiley, N.Y., 1989).

[8] Quantum Chaos, edited by H.A. Cerdeira et al. (World Scientific, Singapore, 1991).

[9] E. Odorico, Nucl. Phys. B 172, 157 (1989).

[10] B.R. Webber, Ann. Rev. Nucl. Part. Sci. 36, 253 (1986).

[11] H.G. Schuster, Deterministic Chaos, 3rd edition (Physik-Verlag, Weinheim, 1995).

[12] R. C. Hwa, Phys. Rev. D41, 1456 (1990); C. B. Chiu and R. C. Hwa, Phys. Rev. D43, 100 (1991).

[13] For a review see R. C. Hwa, in Quark-Gluon Plasma, edited by R. C. Hwa (World Scientific, Singapore, 1990).

[14] C. B. Chiu and R. C. Hwa, Phys. Rev. D45, 2276 (1992).

[15] For a review see E. A. De Wolf, I. M. Dremin and W. Kittel, Phys. Rep. C (to be published).

[16] J. Feder, Fractals (Plenum Press, N. Y. and London, 1988).

[17] A. Białas and R. Peschanski, Nucl. Phys. B 273, 703 (1986); B 308, 867 (1988).

[18] R.C. Hwa, Phys. Rev. D 51, 3323 (1995).

[19] A. Białas and M. Gardzicki, Phys. Lett. B252, 483 (1990).

[20] M. Blazek, Phys. Lett. B247, 576 (1990); P. Duclos and J.-L. Meunier, Zeit. Phys. C64, 295 (1994); I. M. Dremin, Pisma v. ZhETF59 561 (1994); Uspekhi Fiz. Nauk 164, 785 (1994); JETP Lett. 59, 585 (1994). 


\section{Figure Captions}

Fig. 1 A tree diagram for $\mathbf{b}=(1,2,3,3,1)$

Fig. 2 A trajectory of $x_{i}$

Fig. 3 Several possible trajectories.

Fig. 4 Distribution of $i_{\max }$ for QCD and the $\chi$ model.

Fig. 5 Simulated results for the average multiplicities at various generations $i$ for fixed values of $Q / Q_{0}$ indicated by the numbers.

Fig. 6 Simulated results for the normalized variances $V_{i}$ for the same parameters as in Fig. 5.

Fig. 7 Temporal behaviors depicted by $V_{i}$ vs $\left\langle n_{i}\right\rangle$.

Fig. 8 Momentum distribution in terms of $\zeta=\log x$ for QCD branching.

Fig. 9 Momentum distribution in terms of $x$ for the $\chi$ model.

Fig. 10 Momentum distribution in terms of the cumulative variable $X$ for QCD branching.

Fig. 11 Multiplicity distributions in bins of size $\delta=1 / M$ for QCD branching.

Fig. $12 F_{q}$ vs $M$ for $q=2-5$.

Fig. $13 F_{q}$ vs continuous $q$.

Fig. 14 Distributions of $F_{q}^{e}$ calculated event by event.

Fig. 15 Moments of moments $C_{p, q}$ vs $M$ for $p=0.5-2.0$ and $q=2,3,4$. 


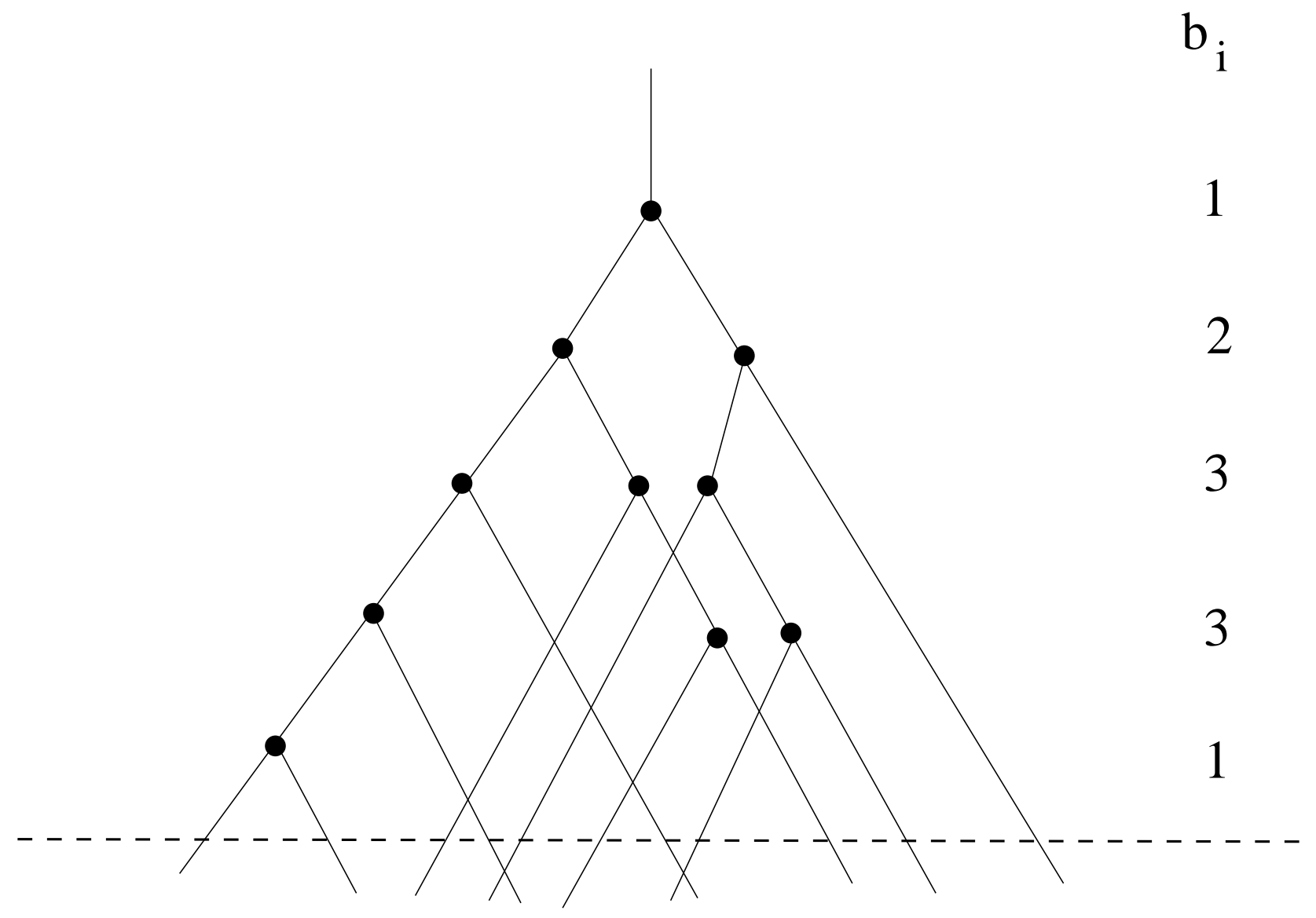




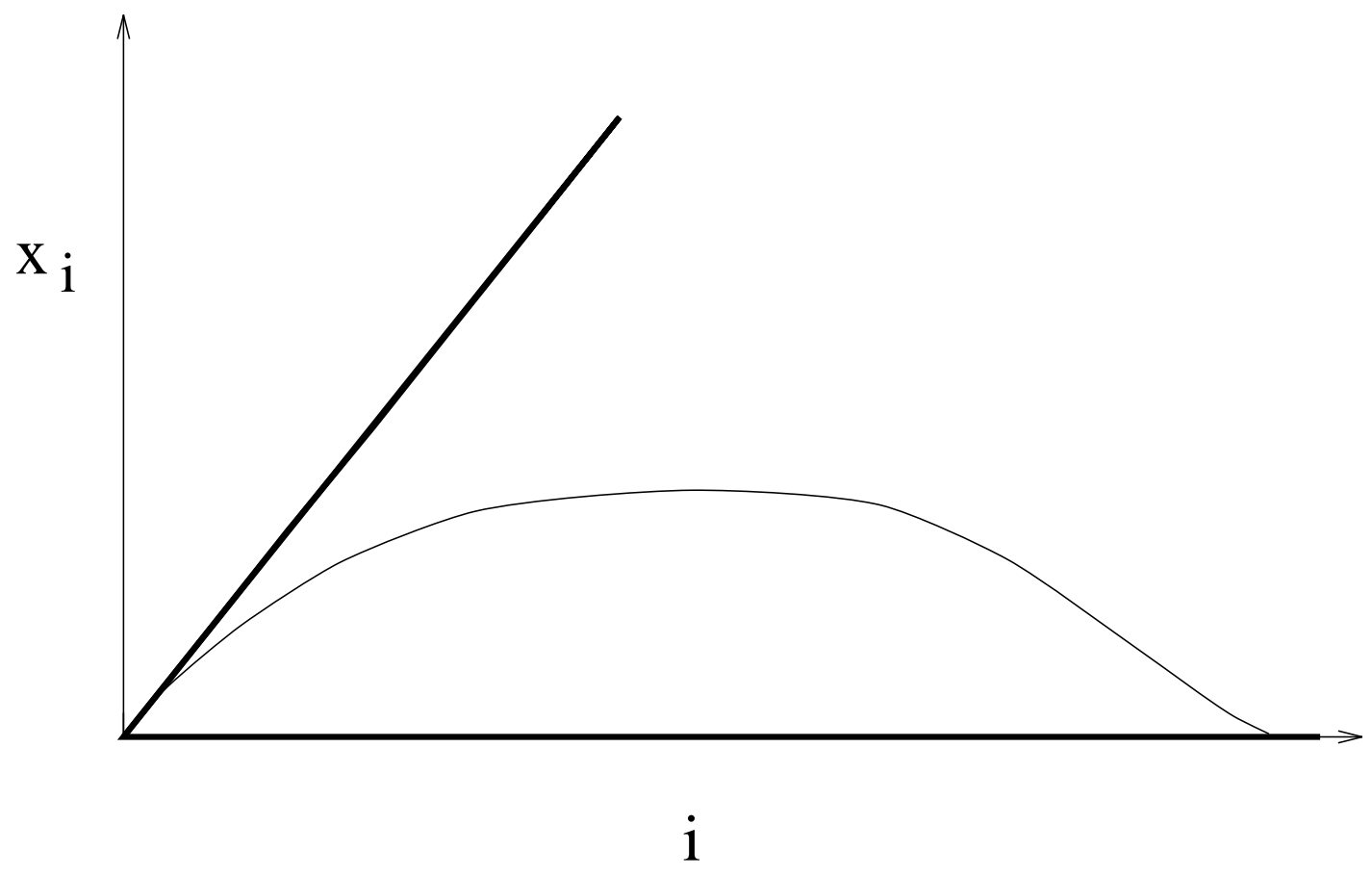




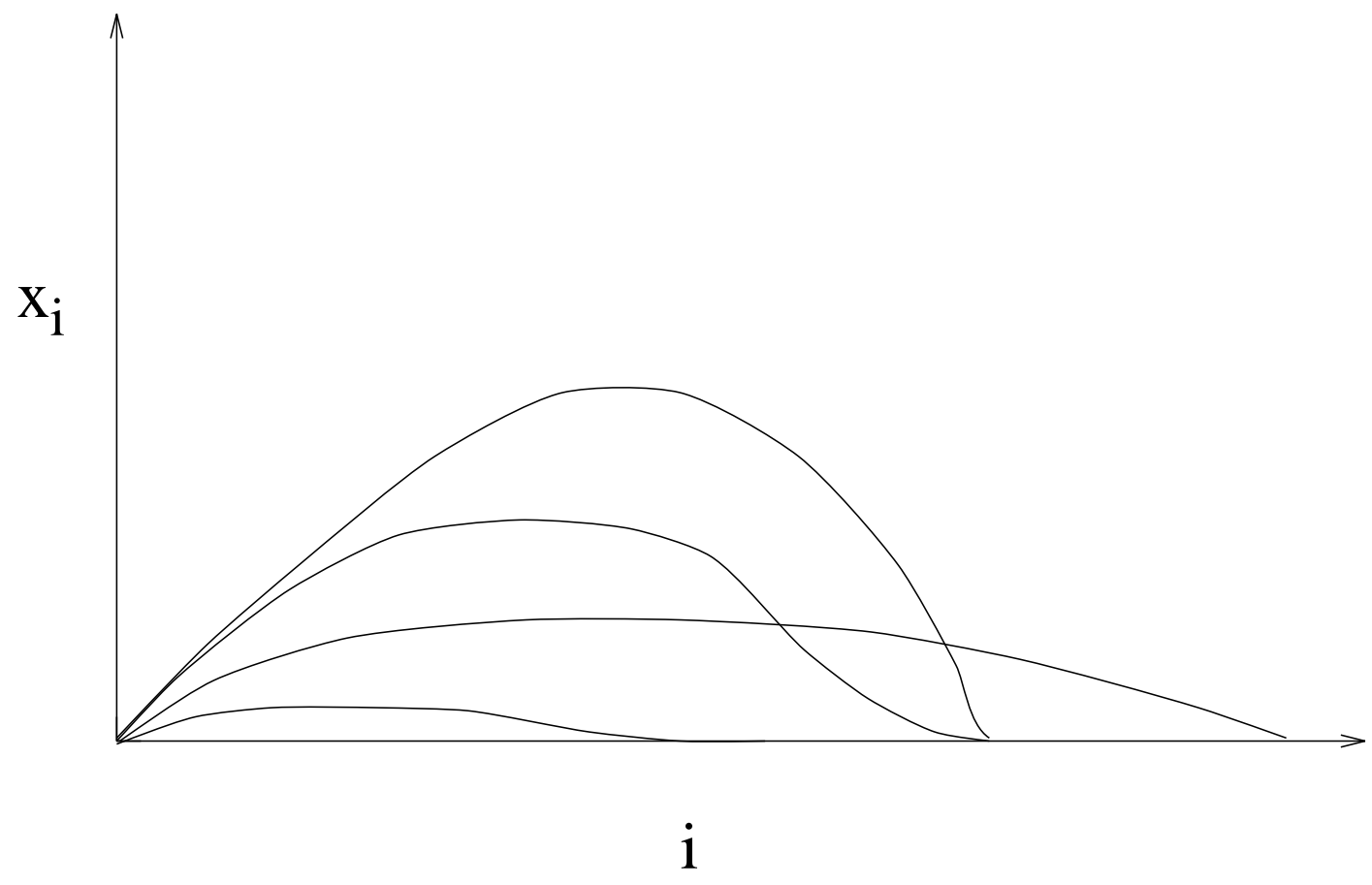




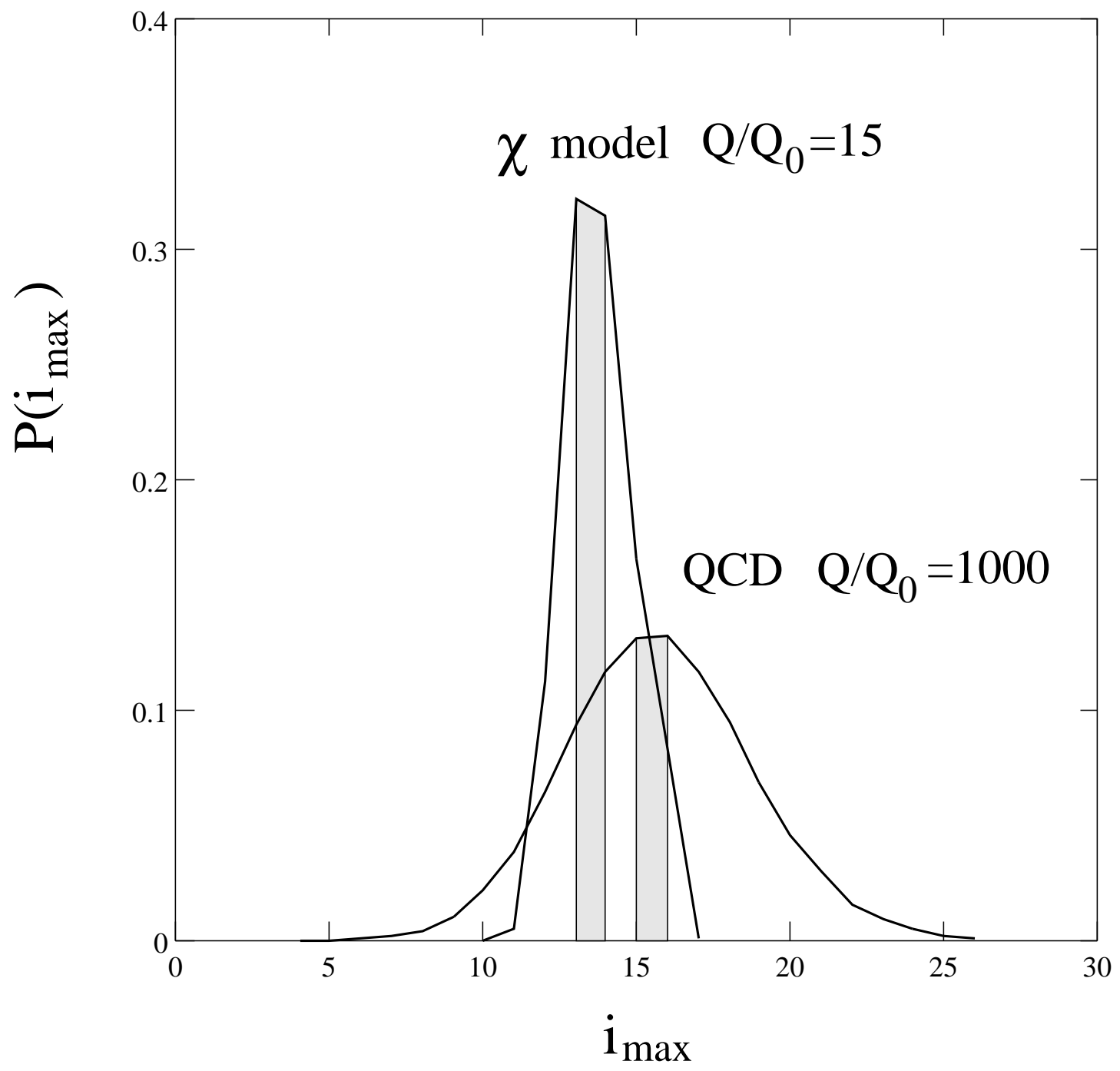




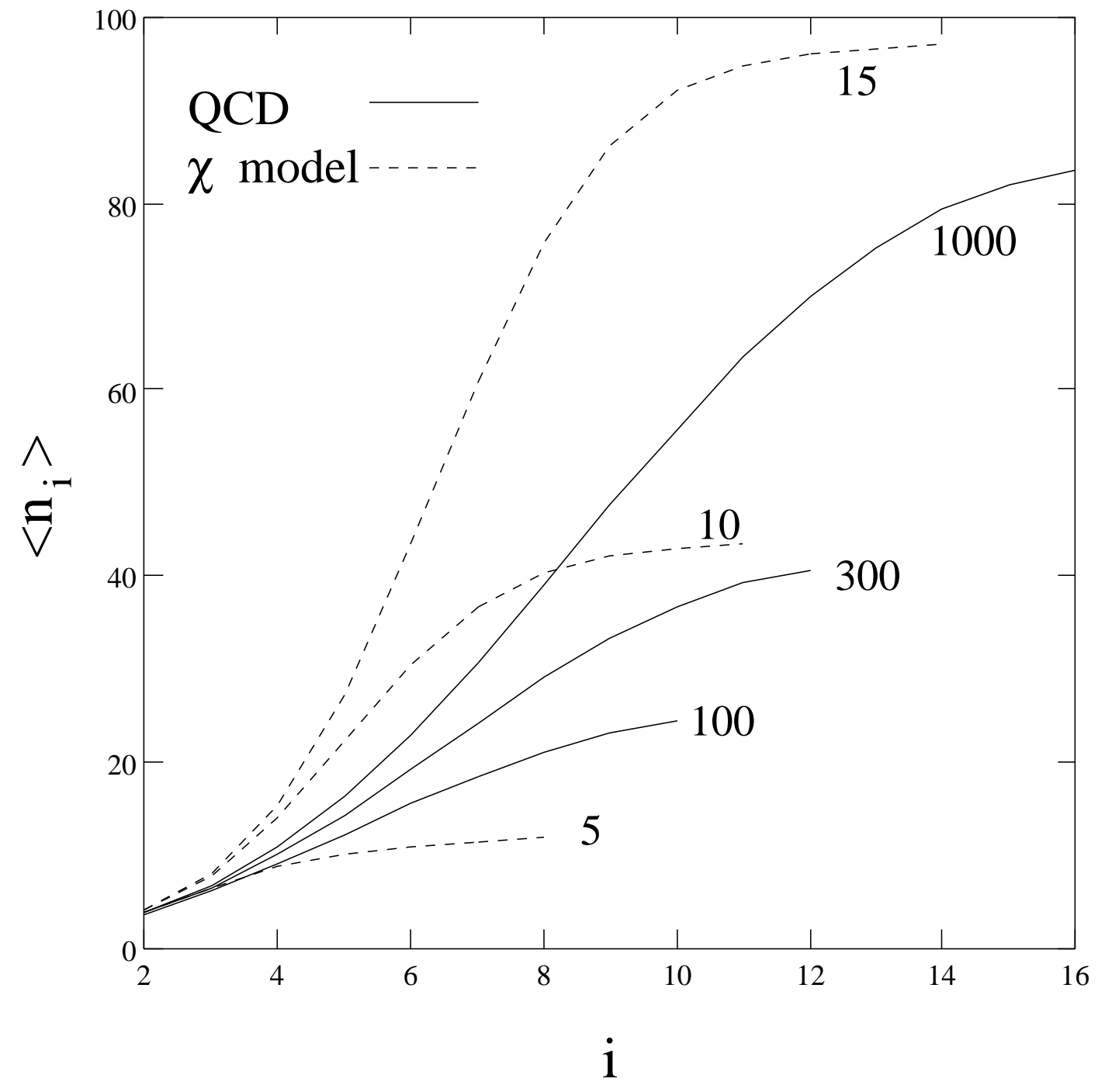




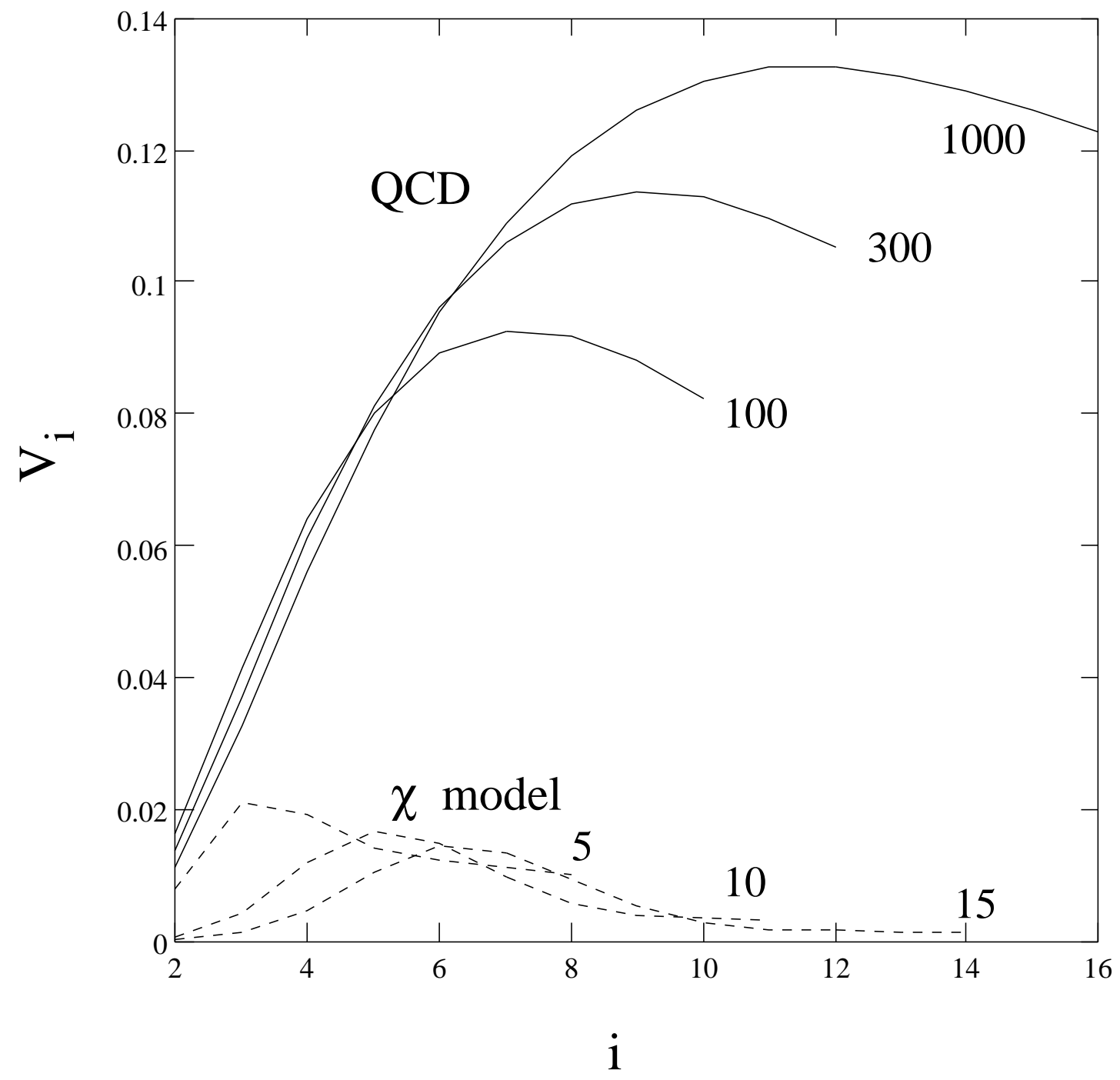




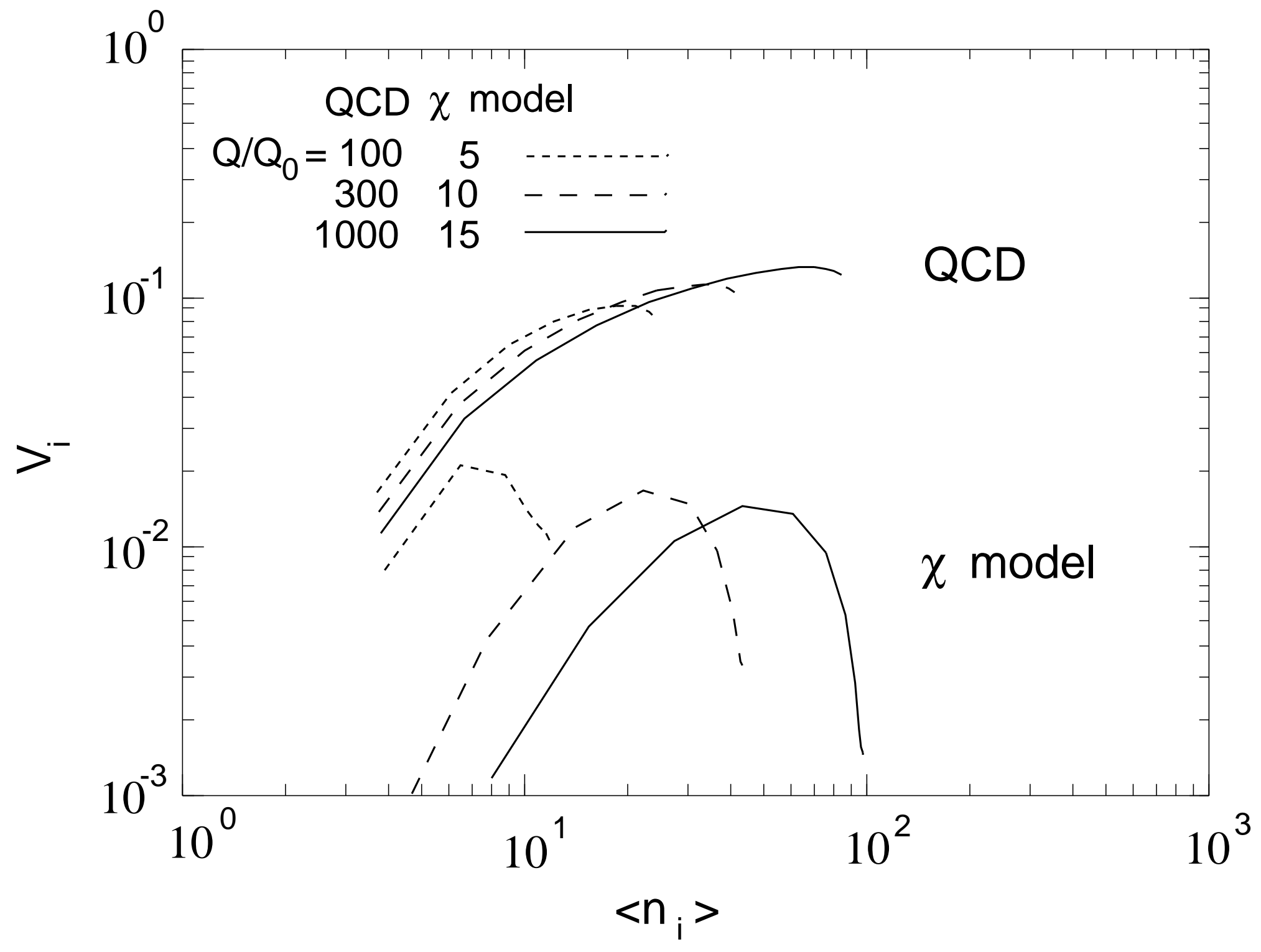




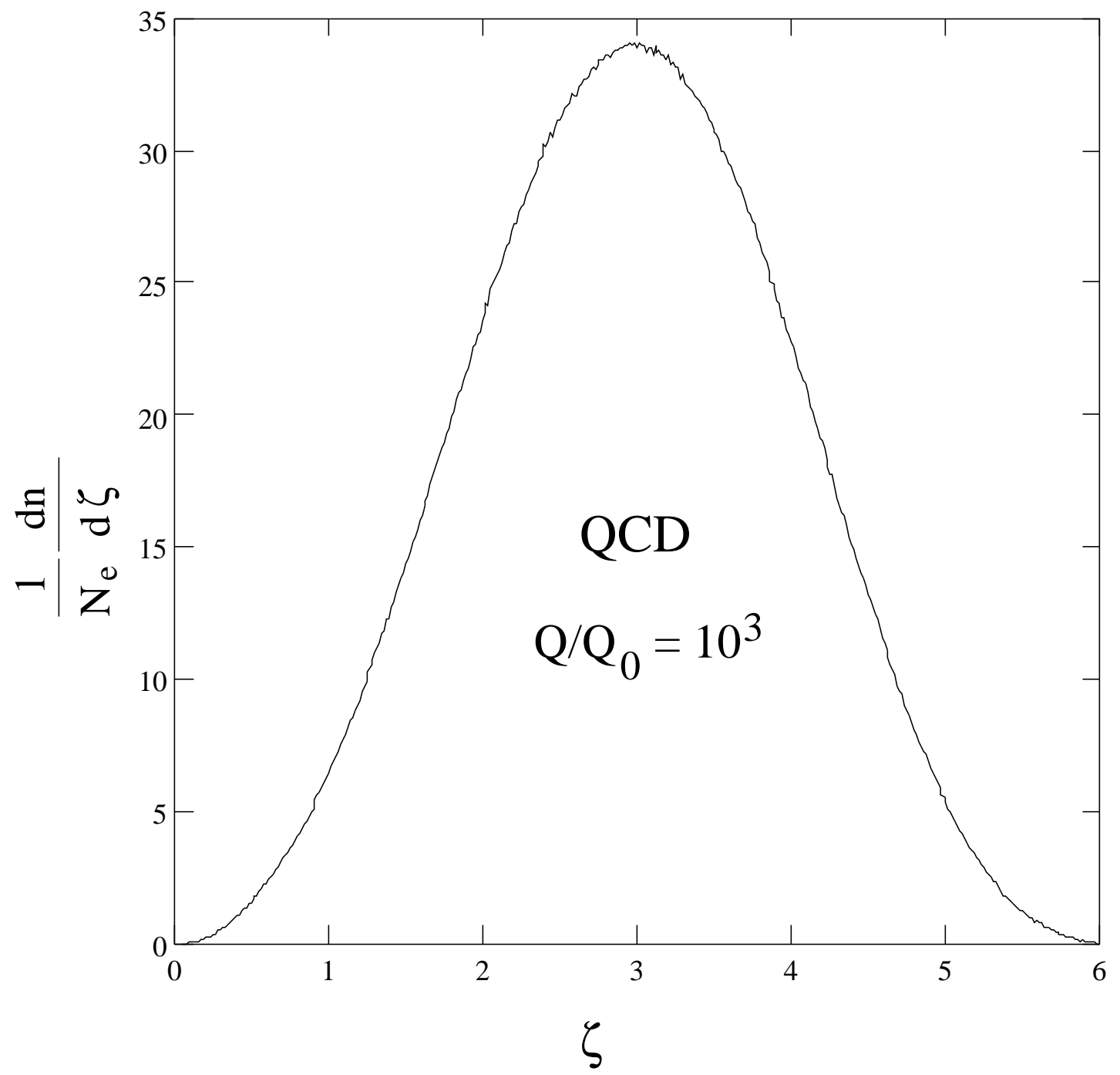




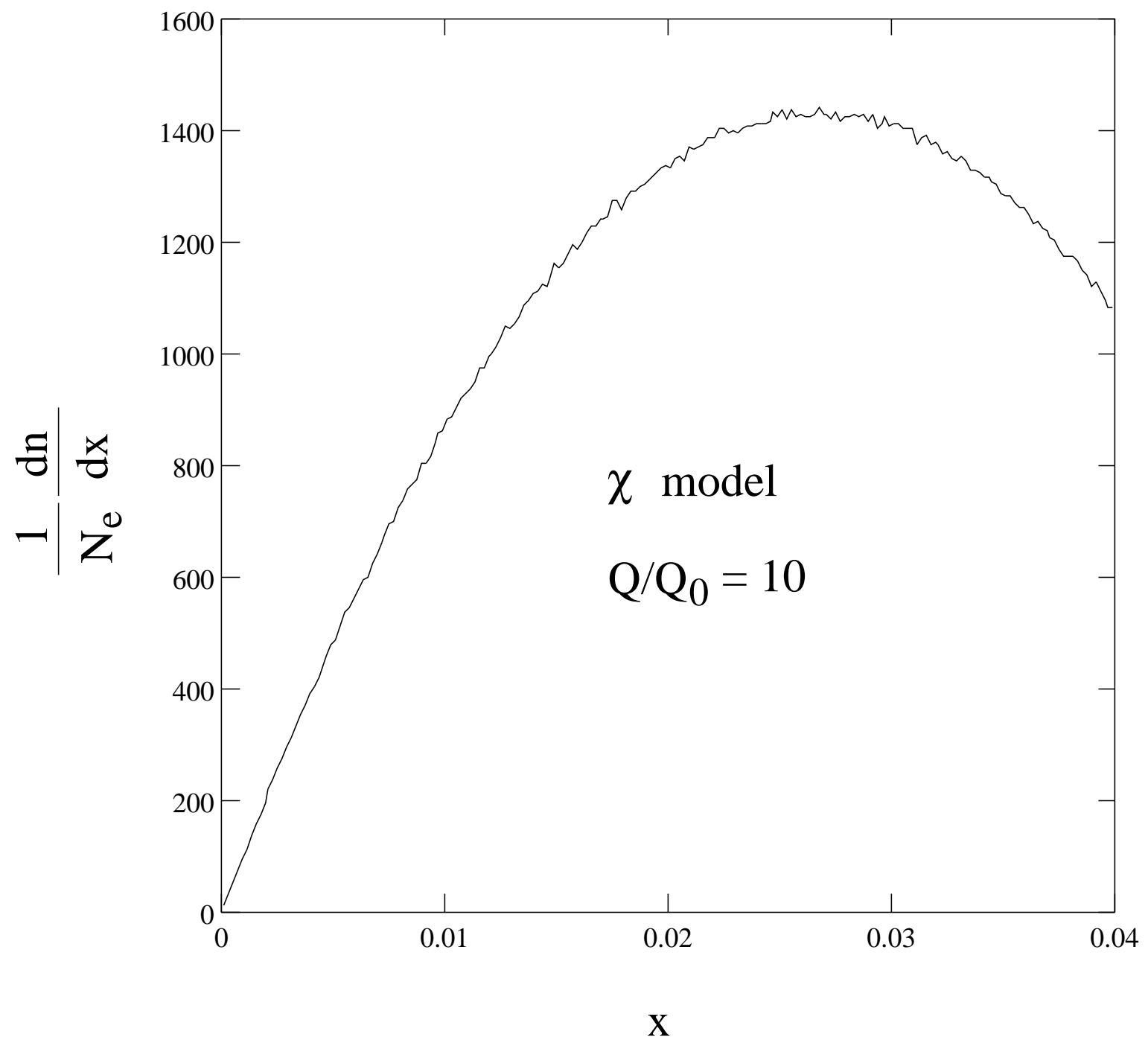




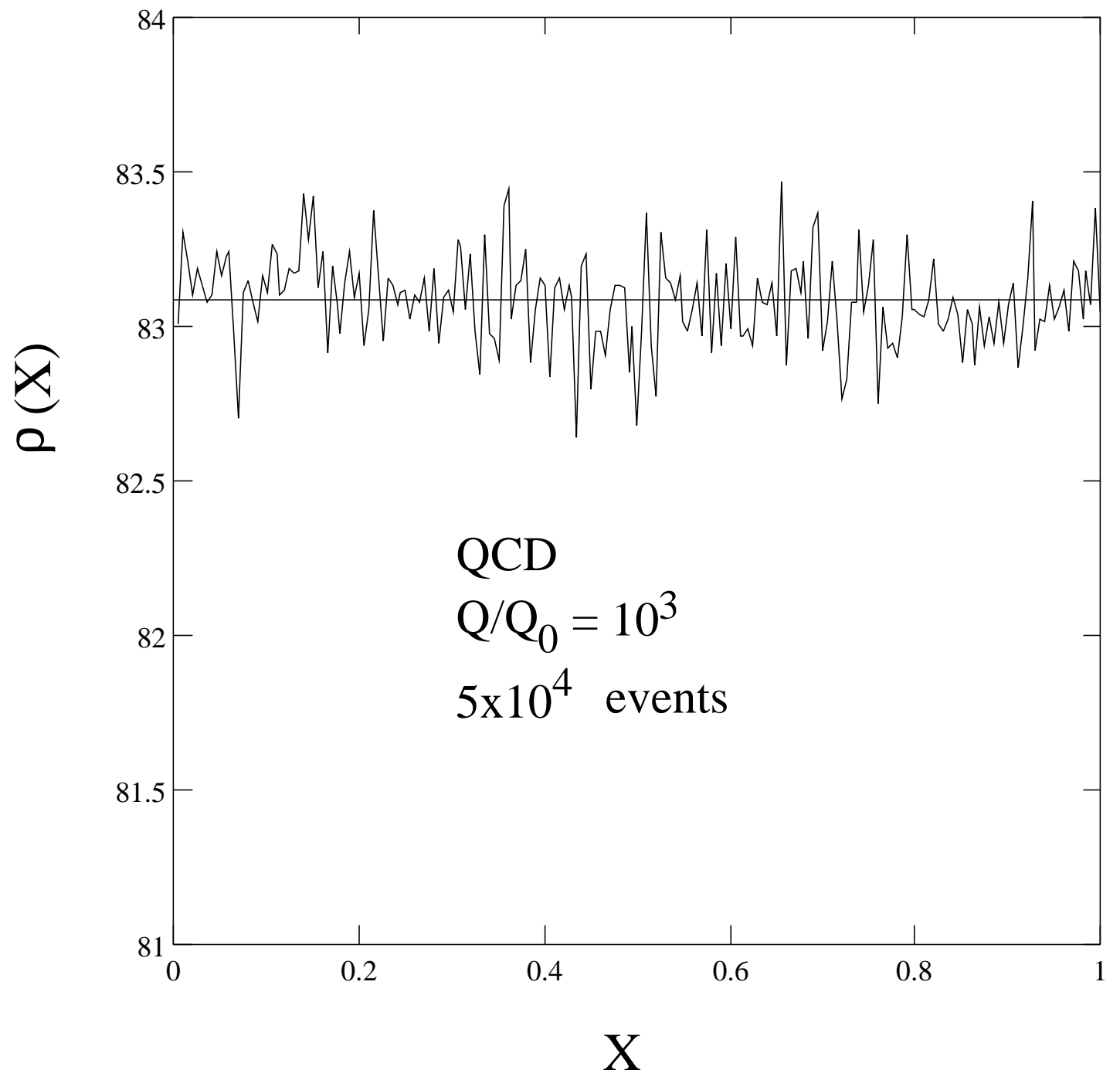




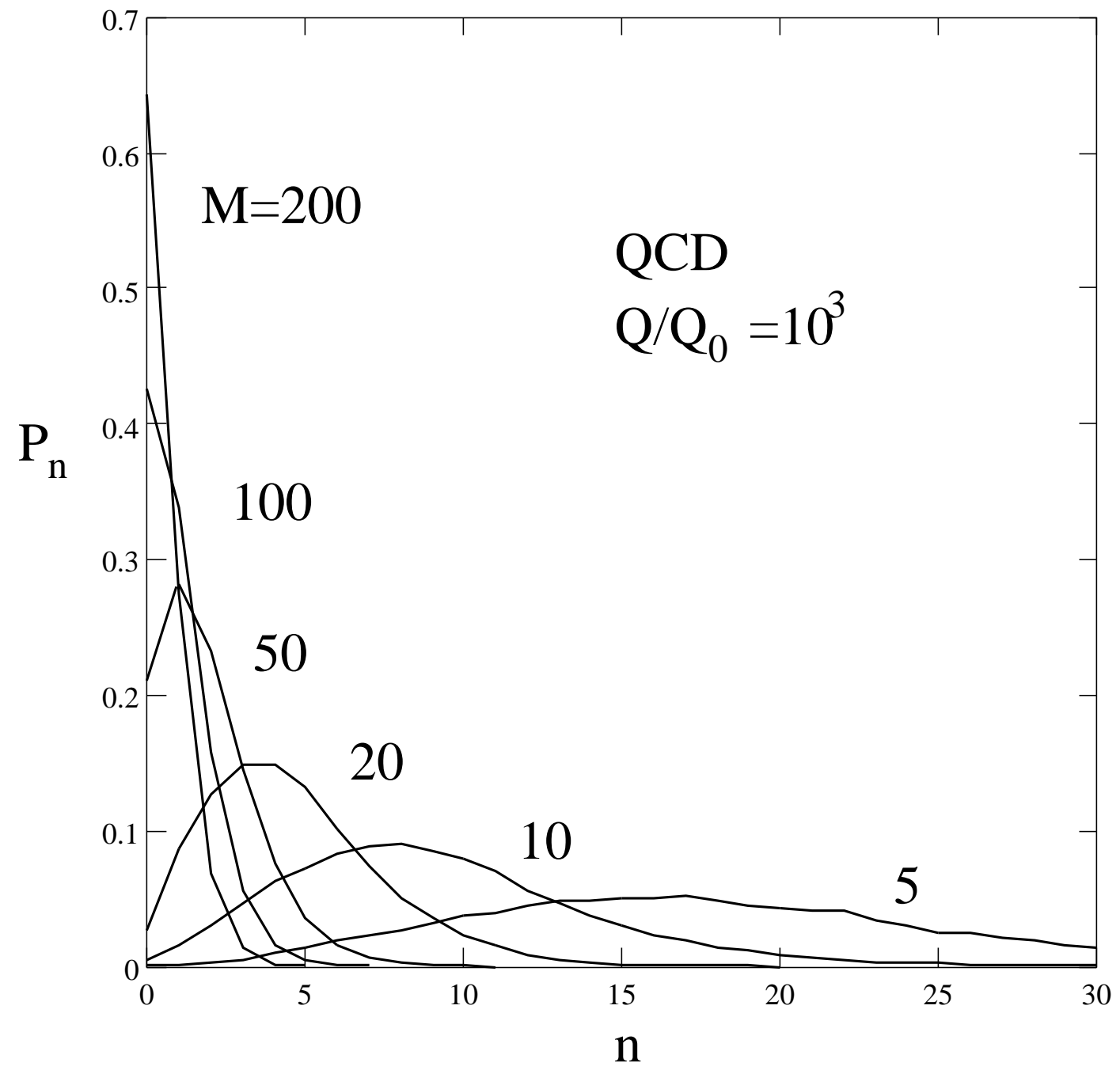




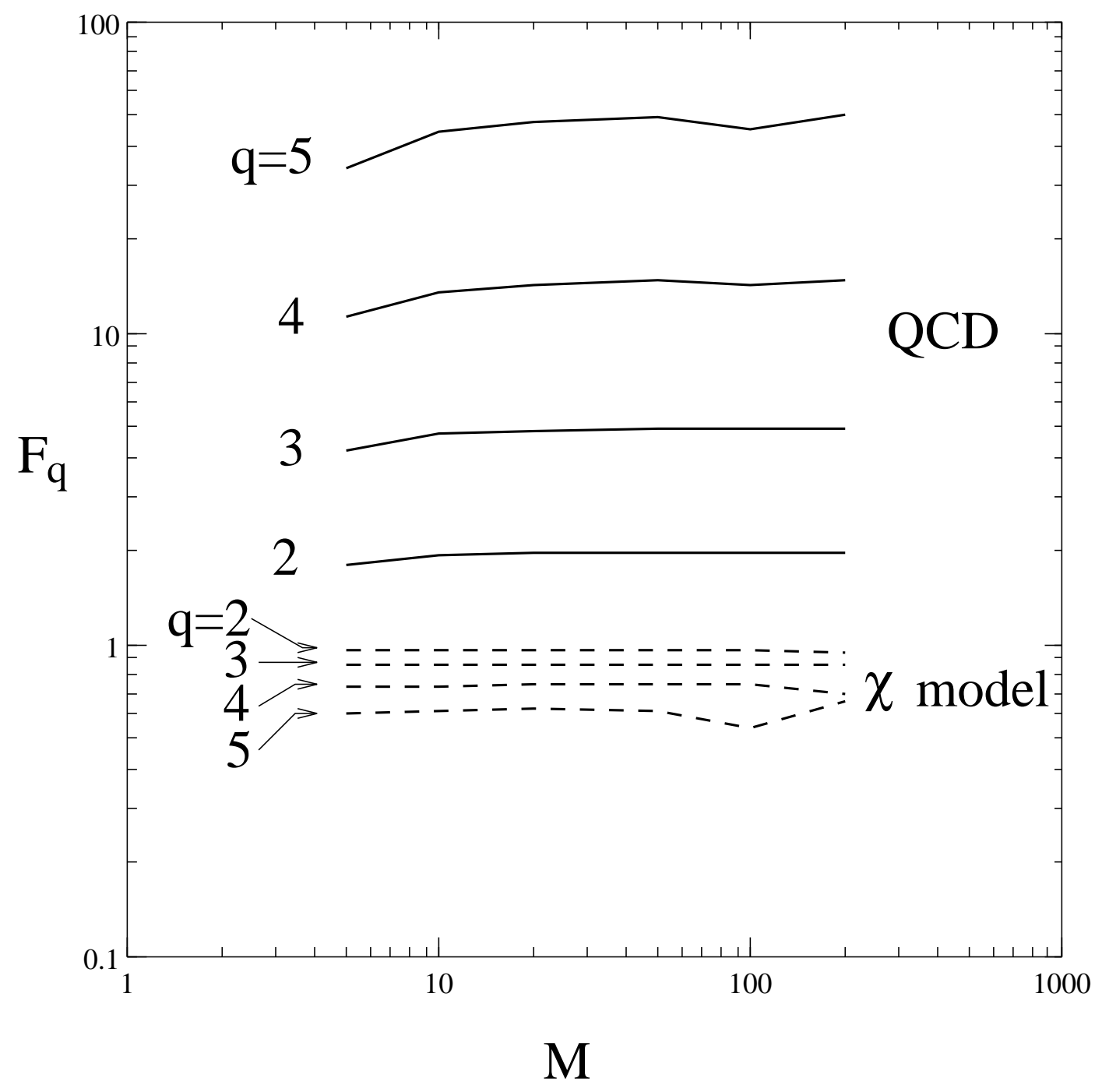




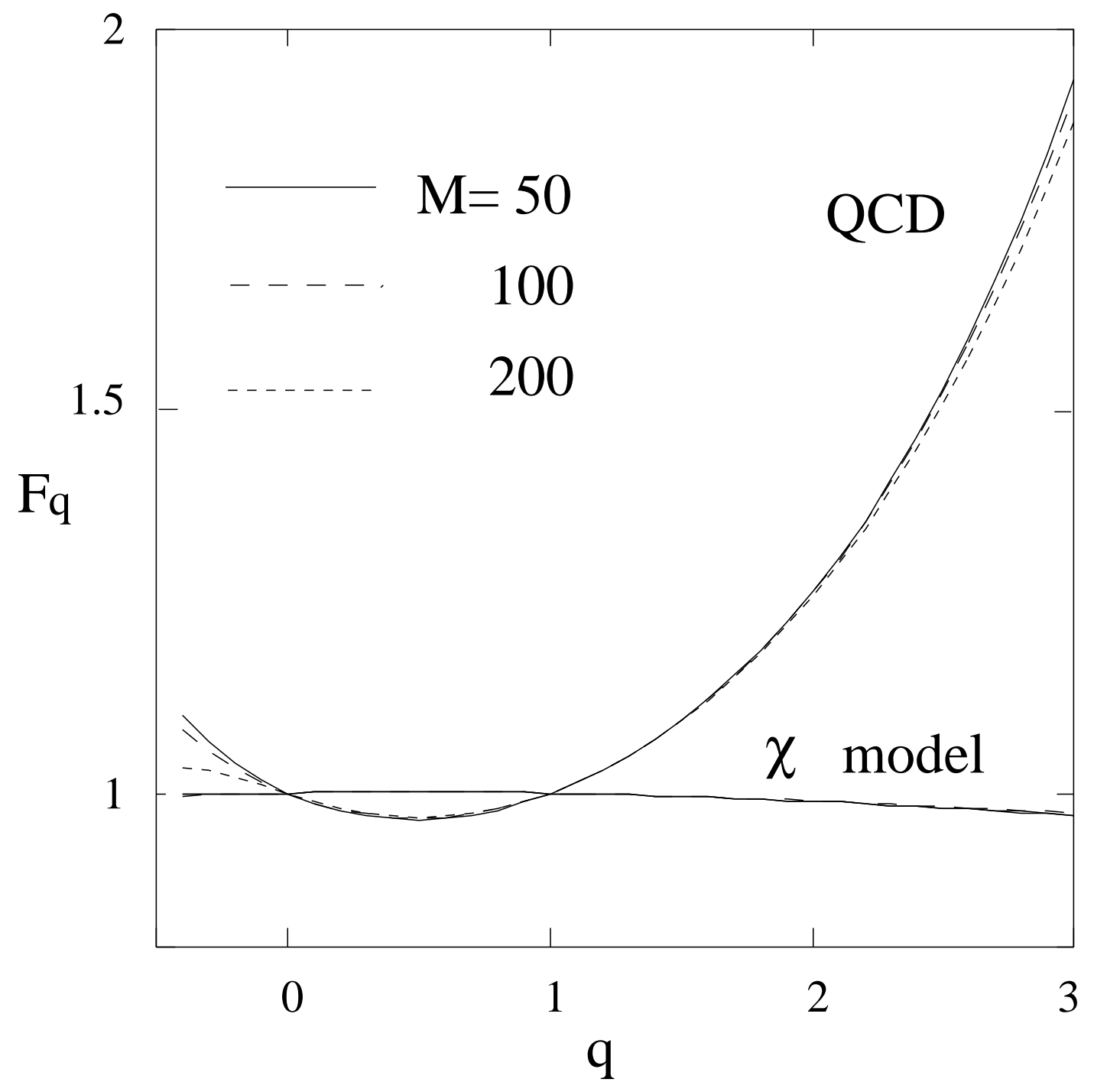




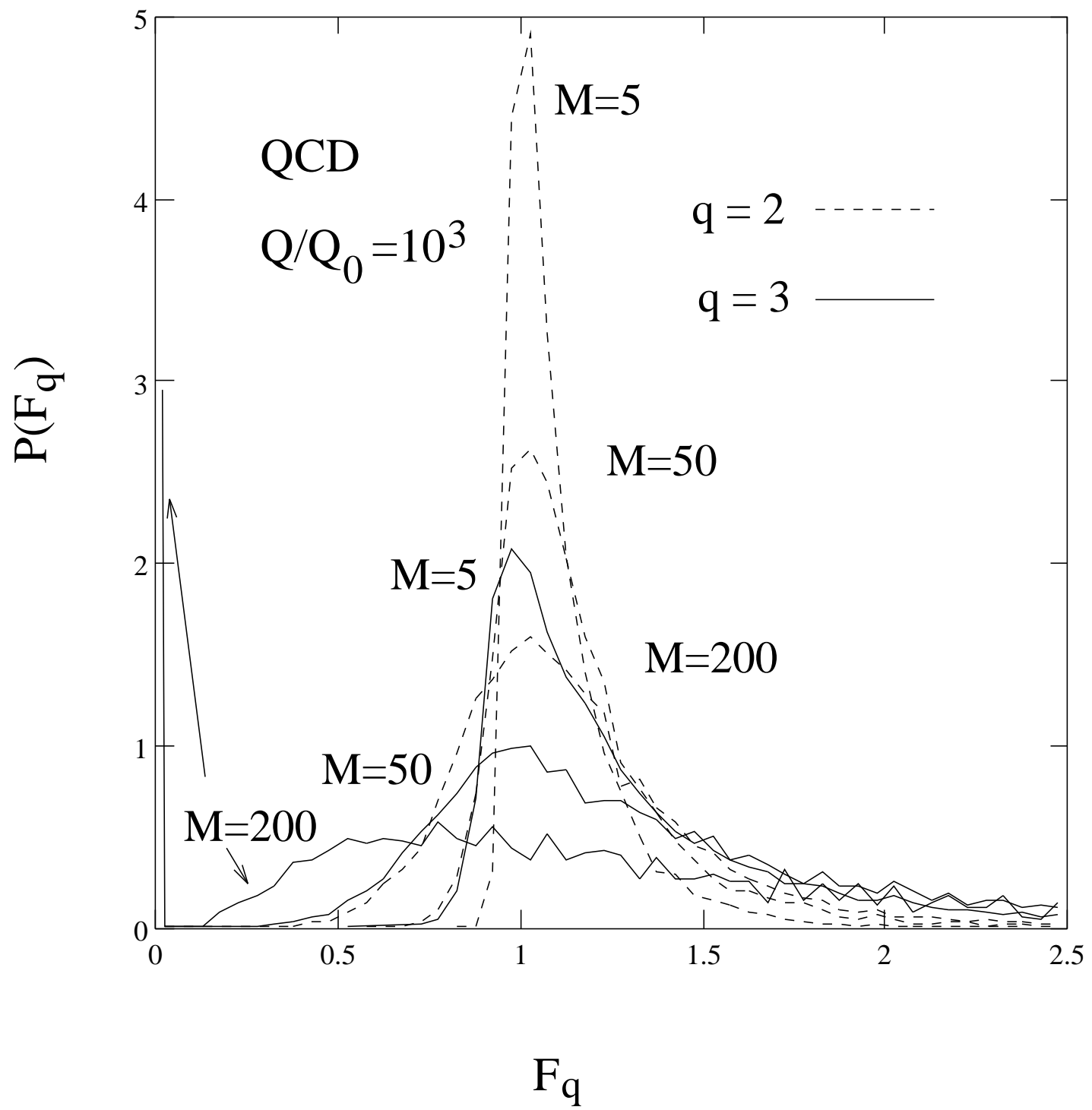




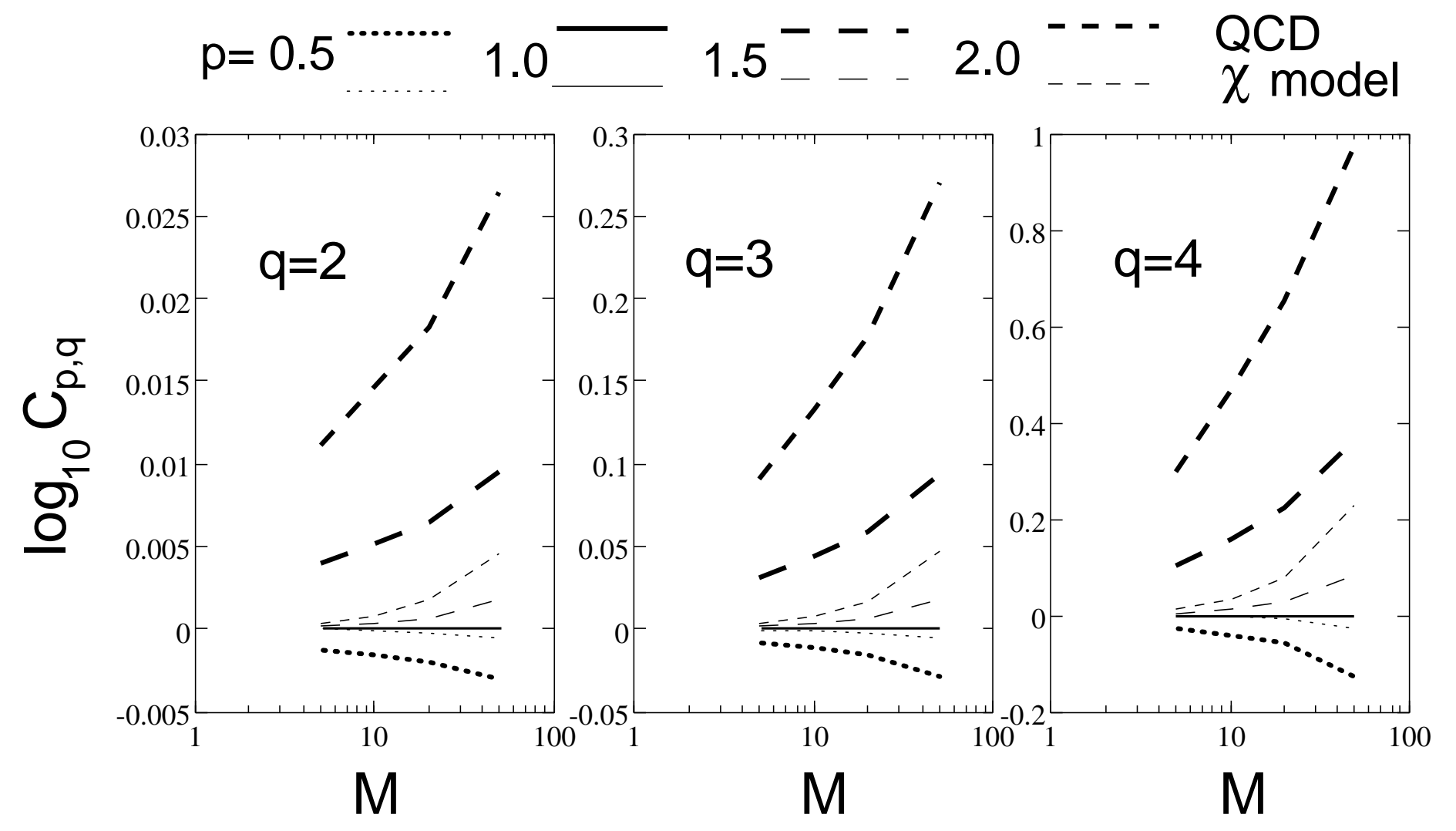

